\title{
PHOTOTHERAPY: MEDICINE FOR THE NEW MILLENNIUM:
}

\author{
Paraskevas P Kontoes $^{1}$, Mario A Trelles ${ }^{2}$ and R Glen Calderhead ${ }^{3}$ \\ 1: Laserlight Clinic for Laser and Plastic Surgery, Athens, Greece; \\ 2: Instituto Médico Vilafortuny, Cambrils, Spain and 3: Japan Phototherapy Laboratory, Tokyo, Japan
}

\begin{abstract}
Phototherapy has been around in clinical practice for more than 5,000 years, but has not shown consistent clinical results or attracted continuous real attention until the last 5 decades, since just before the 'birth' of the laser. Although phototherapy can be construed to mean therapy delivered with any kind of light, including lasers, intense pulsed light (IPL) systems, various incandescent and arc lamps and most recently, light-emitting diodes (LEDs), a gradual consensus has formed that 'phototherapy' means the treatment of a disease or condition with the delivery of athermal and atraumatic levels of photon energy. This rules out IPL systems and laser systems specifically designed for so called nonablative skin rejuvenation, but does not however rule out surgical lasers used in a defocused mode so that the incident power density or irradiance is well below the photodestructive cellular survival threshold. Phototherapy with the new generation of quasimonochromatic LEDs with much greater and more stable output power compared with their earlier generations, is currently attracting a great deal of attention, and indeed controversy, in clinical practice for skin conditions such as acne, skin rejuvenation and wound healing. Pain attenuation has also been reported with a near infrared LED source. It has become evident from the literature that LED therapy works best when two or more wavelengths are combined in a sequential manner, not simultaneously, in a photobiologically appropriate manner for the target cells and tissues. Perhaps an even greater potential for LED therapy exists in its being incorporated into our conventional clinical practice as an adjunctive treatment to improve our already good results, particularly when the cost-effectiveness of these systems is considered. Some of the mechanisms by which this athermal and atraumatic light source can beneficially affect targeted tissues have already been elucidated, but much more work awaits before we can unlock the full scientific potential behind the good clinical results. There is no doubt that LED therapy has arrived, but its impact remains to be maximized.
\end{abstract}

Key words: Light-emitting diodes, acne vulgaris, fibroblasts, mast cells, skin rejuvenation, photobiomodulation, wound healing

\section{Introduction}

\section{Historical background}

Although attracting a great deal of attention at present, phototherapy is not a new concept. The Ancient Egyptians were using sunlight in combination with a topically applied phytophotosensitiser to treat vitiligolike skin lesions some 5,000 years ago. The Greeks

\author{
Addressee for Correspondence: \\ R Glen Calderhead \\ LG Biomedical, \\ 599-3 Kassemba, \\ Tsugamachi, \\ Tochigi-ken, \\ Japan 328-0113 \\ Tel: +81-282-28-0043 \\ E-maIl: docrgc@cc9.ne.jp
}

called phototherapy using sunlight 'heliotherapy', and around $400 \mathrm{BC}$, the 'father of medicine', Hippocrates, actually prescribed heliotherapy for both physical and psychological problems. With the passing of the Ancients and the fall of the Roman Empire, however, the use of heliotherapy sadly fell into oblivion.

Much closer to the present, by the end of the 19th century, red light phototherapy carried out in rooms with red glass windows was commonly used to treat the itchy eruptions of some skin diseases such as rubeolla and rubella, and to treat patients suffering from depressive disorders. Fubini in Italy had already shown that red light increased the metabolism of cells through specific action on the respiratory chain of the cell mitochondria. ${ }^{(1)}$ However a reliable artificial light

Manuscript received: September 2006

Accepted for publication: Octorber 2006 
source was not available until the Danish physician and scientist Niels R Finsen developed his arc lamp which was capable of delivering a wide waveband of ultraviolet (UV) visible and infrared (IR) components. In the mid 1950's the excess of bilirubin in neonates, until then frequently fatal, started to be treated successfully with blue light phototherapy.

The genesis of the laser and light-emitting diodes

Einstein expounded his quantum theory in 1904, on which much of the basics of stimulated emission of radiation (the 'SER' of 'LASER') were based. In 1960, Professor Theodore Maiman successfully constructed the first practical laser device, based on a solid state doped ruby crystal, which delivered a pulse of extremely pure visible red light energy so powerful that it could drill a tiny hole through a stack of razor blades. Between then and the mid 1960's most of the mainstay lasers currently used in surgery and medicine were developed, including the carbon dioxide (CO2), argon, helium neon (HeNe), neodymium yttrium aluminium garnet (Nd:YAG), holmium YAG (Ho:YAG) and erbium YAG (Er:YAG) lasers. The laser diode was also developed in this era, as was the first commercially-available visible noncoherent light light-emitting diode (LED) (Holonyak, General Electric Company).

\section{Evolution of 'phototherapy'}

Strictly speaking, the application of any device that produces light which can be used in surgery and medicine to treat patients can be classed as 'phototherapy'. However, a gradual distinction has been drawn between destructive and invasive photosurgery, based on deliberately delivered thermal damage, and minimally- or noninvasive phototherapy, based on athermal energy exchange between incident photons and target cells. That latter application comprises the central theme of this article.

The early adopters of $\mathrm{CO} 2$ laser surgery reported that they were getting better results, faster wound healing, less oedema and less postoperative pain with the laser that when the same procedure was performed with the conventional scalpel or with electrosurgery. This was first termed the alpha-effect, ${ }^{(2)}$ and then classified as simultaneous low reactive level laser therapy. ${ }^{(3)}$ These reports showed clearly that there was some 'extra' component associated with the 'L' of laser (i.e. 'light') which took the aftermath of laser surgery beyond a mere thermal reaction. When a surgical laser beam, such as that from a CO2 laser, is aimed at tissue, a series of both photothermal and athermal photoreactions occurs almost simultaneously down into the tis-

Fig. 1: Schematic showing the range of thermal (with approximate temperature ranges) and athermal reactions when a surgical laser beam is incident on the target tissue, depending on the wavelength and tissue type. All reactions occur almost simultaneously, and the photobiomodulation zone is present even is surgical laser excision, incision or vaporization.

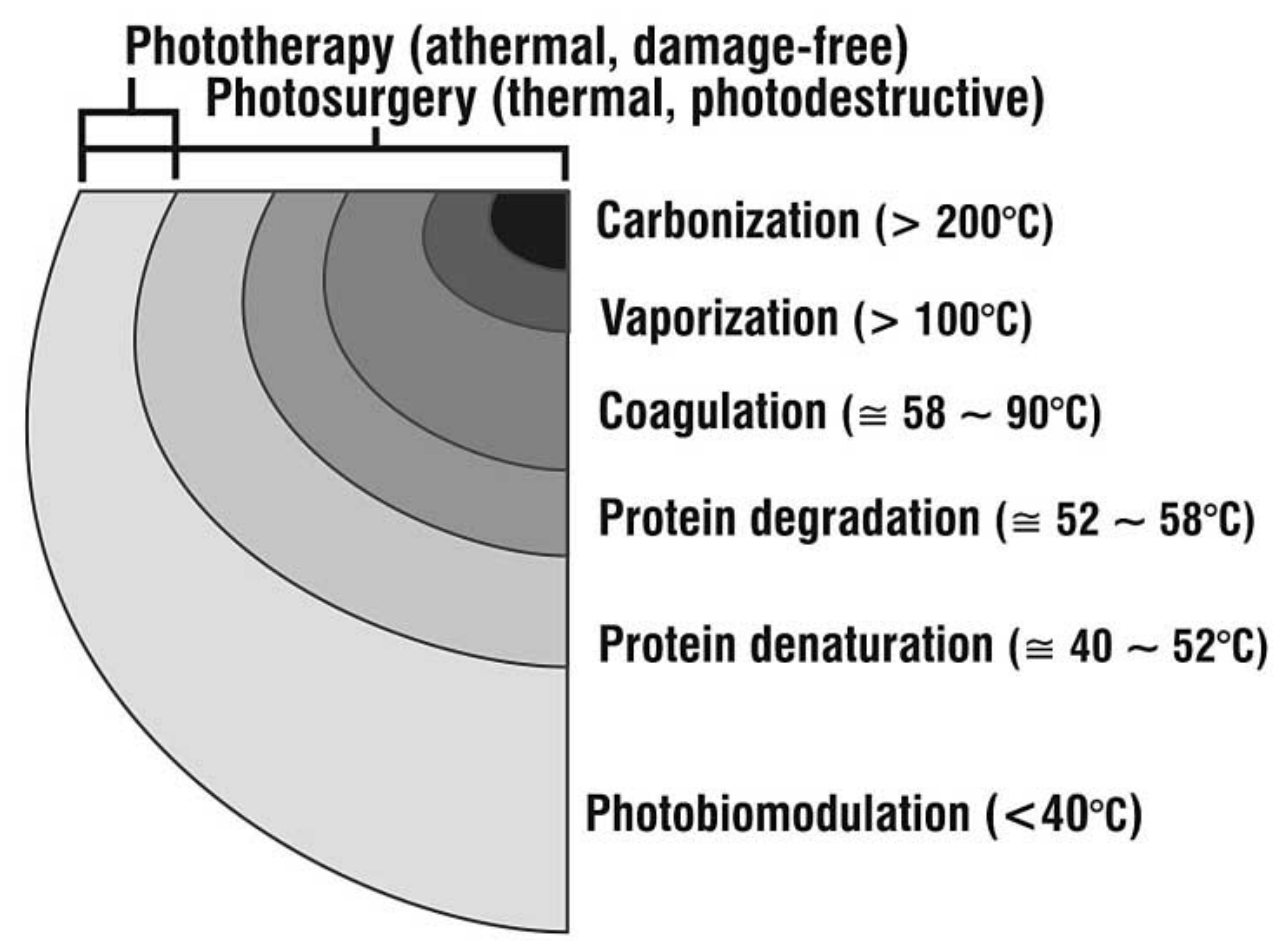


sue, as ever-decreasing levels of photon intensity occur in well-demarcated, roughly concentric zones from the total destruction seen in the vaporisation and coagulation zones: these occur through the partial destruction in the protein degradation and denaturation zones, to the normal-appearing tissue architecture in the outermost zone, the photobiomodulation zone. (Fig. 1)

In the outermost zone of athermal photobiomodulation, the skin cells in the target tissue not only remain alive and unharmed, but have received an energy 'boost' by the direct athermal exchange of energy between the incident photons and the cells or more accurately their subcellular components, since energy can never be lost. This increased energy level in the cells can result in several different reactions: injured or compromised cells can be repaired; mitotic cells can be induced into a higher rate of replication; or cells with a specific function perform their allotted task faster and in a more efficient manner. ${ }^{(4)}$ It is the action of these photobiomodulated cells on the irradiated tissue, such as the dermal matrix and epidermal basal layer, which assures good wound healing, faster evolution of oedema and erythema, and pain control. Without the influence on the evolution of repair and regeneration exerted by the photobiomodulation zone, i.e. the phototherapeutic zone, the zones of thermal damage in the upper layers of the tissue as seen in Figure 1 might as well be created with a red-hot poker or a household iron. The nature of true phototherapy is certainly not photothermal. ${ }^{(5)}$

\section{Phototherapy sources}

Given that phototherapy should be nonthermal and damage free, this rules out surgical laser ablative resurfacing, or intense pulsed light (IPL) systems and nonablative skin rejuvenation lasers as phototherapy sources, since these latter systems depend for their desired clinical effect on the deliberate delivery of a zone of controlled thermal damage below an intact epidermis. This does not mean that surgical lasers cannot be used to deliver low photon intensities which have an athermal action on the target tissue. One of the earliest reports on the analgesic effects of laser energy in 1981 compared a defocused Nd:YAG laser compared with a gallium aluminium arsenide (GaALAs) dedicated laser therapy system. ${ }^{(6)}$ Defocused surgical lasers are thus perfectly capable of delivering phototherapeutic doses: a $20 \mathrm{~W}$ CO2 beam, normally capable of very precisely excising and vaporizing large amounts of tissue, when delivered over an area $10 \mathrm{~cm}$ in diameter gives a power density of around 800 $\mathrm{mW} / \mathrm{cm}^{2}$, which is well below the power densities associated with even mild tissue heating.

Surgical lasers are, however, rather expensive and bulky tools to be used simply for phototherapeutic applications, are associated with potential safety hazards, and require the provision of strictly controlled treatment environments. Laser therapy systems, on the other hand, while still rather expensive, are designed for phototherapeutic indications, delivering as they do a true monochromatic beam of one single wavelength. However they have one major drawback which is the very small treatment area per shot. Treating a large area, such as a full face in the photorejuvenation of photodamaged skin, thus becomes very time consuming and rather therapist-intensive.

Up till the end of the 1990's, light-emitting diodes (LEDs) were totally unsuitable for phototherapy: despite being very bright and very inexpensive, they gave very unstable output powers, were extremely divergent and had very large wavebands, 100 or more nanometres $(\mathrm{nm})$ either side of the rated wavelength. Cells are very sensitive targets, and chromophores, the target molecules in cells for phototherapy, are highly wavelength-dependent and thus require very narrowband light to activate them. In the late 1990's, Whelan and colleagues working in the Space Medicine program in the United States National Aeronautic and Space Administration (NASA) developed a new generation of super-powerful, quasimonochromatic LEDs, which very quickly proved useful in the clinical field, including wound healing. $(7,8)$ The LED thus presented clinicians with an ideal light source for phototherapeutic applications, as LEDs are capable of being mounted in adjustable planar arrays, arranged on articulated arms, to deliver quasimonochromatic light of clinically useful photon densities over large areas of target tissue, and in a hands-off manner.

\section{Phototherapy in Clinical Practice}

Many clinical applications for LED therapy have been established in the last few years, and more continue to appear with each major national and international congress. The genesis of LED therapy was the use of red light at $633 \mathrm{~nm}$ (the same wavelength as the historically well-reported helium neon [HeNe] laser) to activate exogenously-applied aminolaevulunic acid (5-ALA) in the treatment of non-melanoma skin cancers and actinic keratoses. (9) A $633 \mathrm{~nm}$ LED-based system with adjustable LED panels on an articulated arm was specifically developed for this application by a UK company (Omnilux PDT®, Photo Therapeutics, Fazeley, UK) which has had excellent and robust results. (Figs. 2, 3) 


\section{ORIGINAL ARTICLES}

Fig. 2: Basal cell carcinoma (a) before and (b) 6 months after $633 \mathrm{~nm}$ LED PDT therapy with 5-aminolaevulinic acid. 20\% ALA, 2 hrs occlusion. Red LED $633 \mathrm{~nm} .20 \mathrm{~min}, 96 \mathrm{~J} / \mathrm{cm}^{2}$ Good result, good cosmesis, no recurrence.
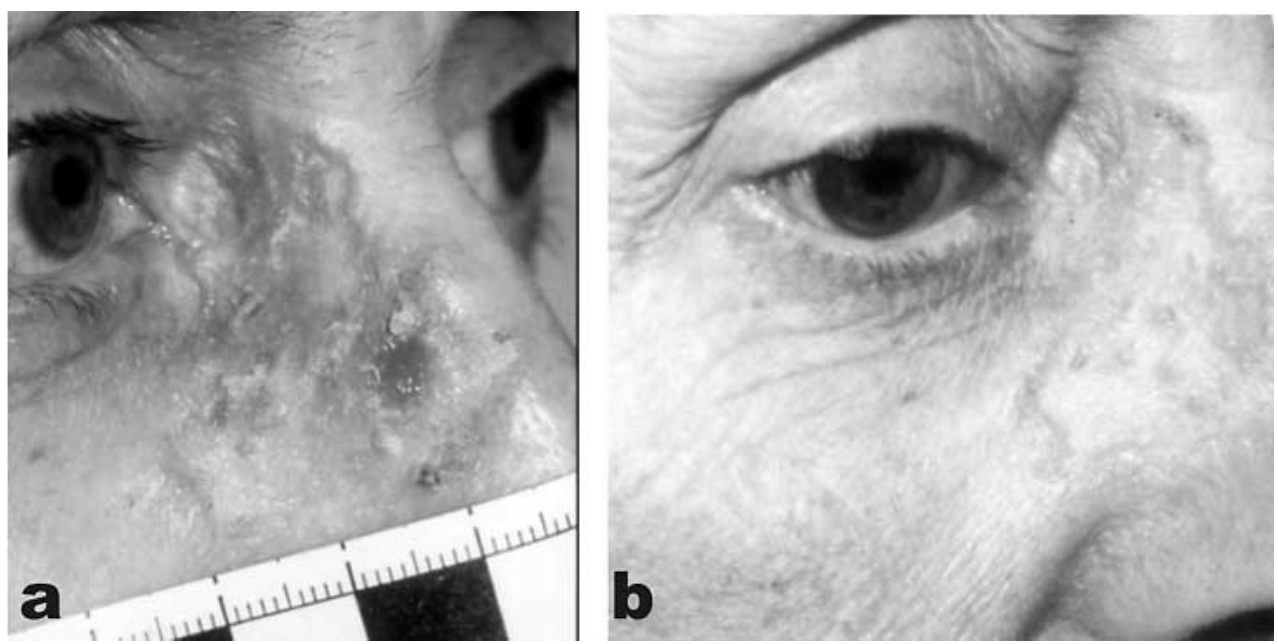

Fig. 3: Actinic keratosis (a) before and (b) 6 months after 633nm LED PDT therapy with 5-aminolaevulinic acid. 10\% ALA, $30 \mathrm{~min}$ occlusion. Red LED $633 \mathrm{~nm} .20 \mathrm{~min}, 96 \mathrm{~J} / \mathrm{cm}^{2}$ Good result, no recurrence, patient very happy.
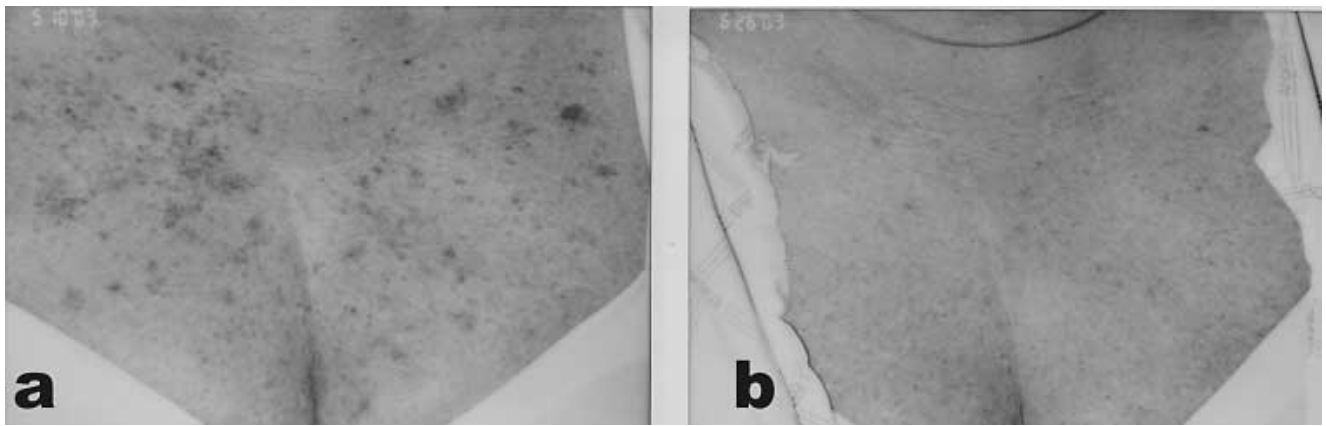

Fig. 4: $633 \mathrm{~nm}$ LED 5-ALA PDT for acne vulgaris in a $23 \mathrm{yr}$ old male. (a): Before; and (b): After 5\% 5-ALA PDT with Omnilux Revive, 633 nm, 20 min 126 J/cm². (Courtesy Of Dr Kanno, Nishiarai Skin Clinic, Tokyo, Japan)
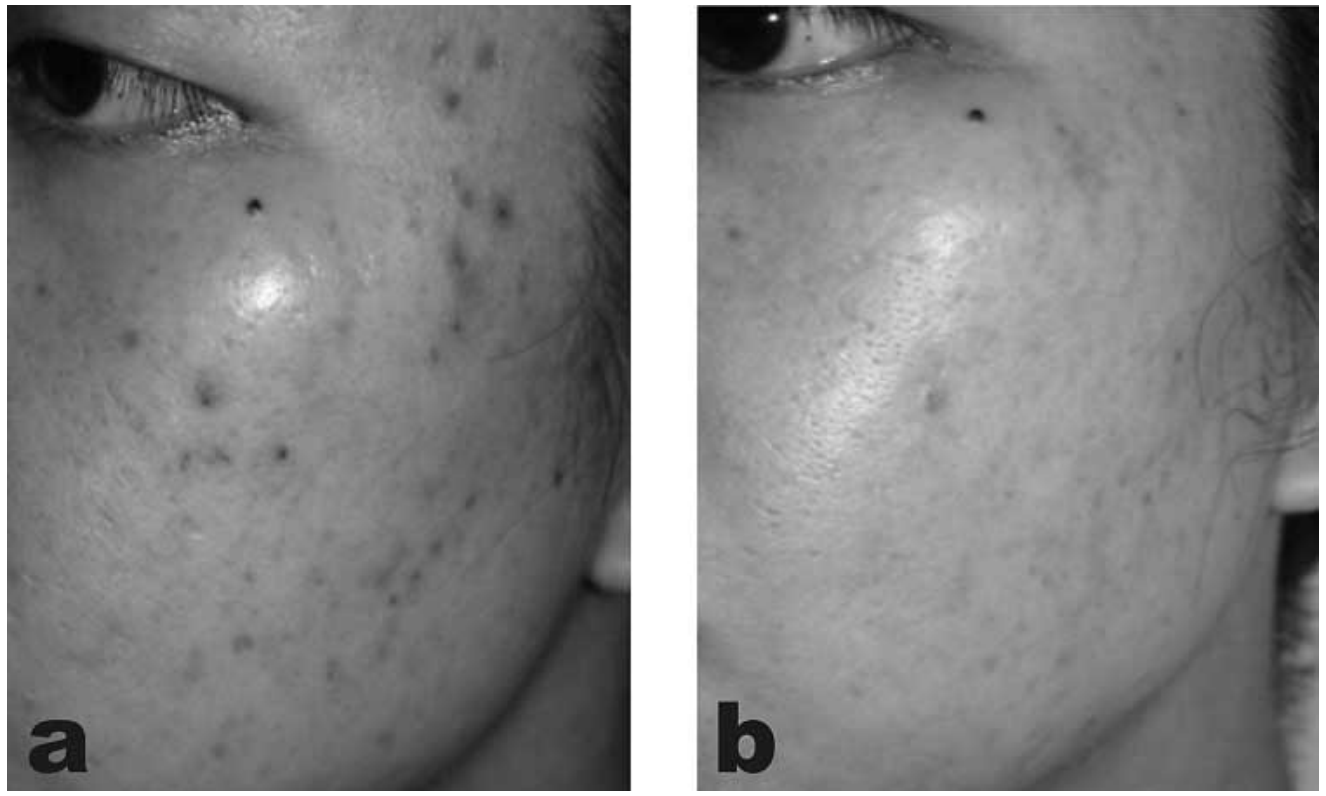
Fig. 5: Action spectra of Pp IX and Cp III showing a small peak at $633 \mathrm{~nm}$, but a peak some orders of magnitude bigger at $415 \mathrm{~nm}$, in the visible blue.

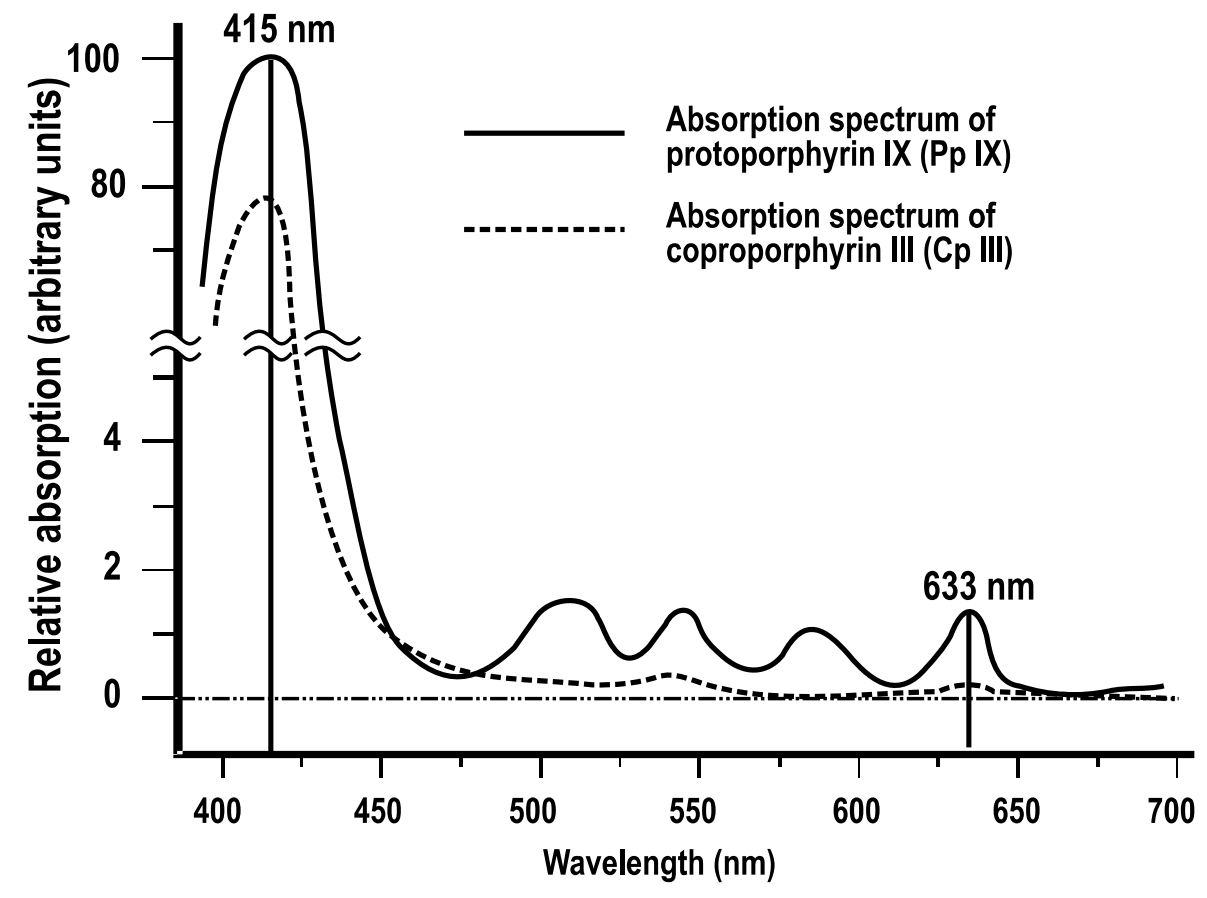

\section{Acne Vulgaris}

Moving away from the more serious skin lesions, this treatment combination then spread to the successful treatment of acne vulgaris with much lower concentrations of 5-ALA and much shorter incubation times, and a more powerful $633 \mathrm{~nm}$ LED source (Omnilux revive $\left.{ }^{\circledR}\right)$ (Fig. 4). ${ }^{(10)}$ Red LED therapy in combination with 5-ALA PDT was also tried for skin rejuvenation, ${ }^{(11)}$ another fast-emerging dermatological application for phototherapy, and addressed in more detail below.

The mechanism of action for red light PDT with 5-ALA is the conversion in the haeme cycle of the exogenous 5-ALA, which has penetrated into the skin, to a porphyrin (protoporphyrin IX, Pp IX) which builds up in quantity in the skin of the region of interest, and whose action spectrum contains a peak at 633 $\mathrm{nm}$. An appropriate dose of $633 \mathrm{~nm}$ light activates the Pp IX and results in the very transient production of reactive oxygen species (ROS) which causes oxidative stress-induced apoptotic cell death in the affected cells. Because the reaction is only a few nanoseconds long, the damage is limited to the targeted cells. The treatment is, however, painful and can result in a severe inflammatory response and patient down-time.

This 'no pain, no gain' approach may be accept- able in curing serious cancerous and precancerous skin lesions, but is it acceptable in acne, or especially in skin rejuvenation? Fig. 5 illustrates the absorption spectrum of Pp IX and another porphyrin, coproporphyrin III (Cp III). In addition to the comparatively small peak at $633 \mathrm{~nm}$, Pp IX, in common with Cp III, has its largest peak in the visible light spectrum very much at $415 \mathrm{~nm}$. It was postulated that, as both these porphyrins are present in active propionibacterium acnes ( $P$. acnes) which is the bacterium mostly responsible for implementing the inflammatory cycle in acne vulgaris, exogenous application of 5-ALA might not be required if a lamp could be developed at $415 \mathrm{~nm}$ to achieve endogenous PDT of the naturally-occurring $P$. acnes-located porphyrins. The only LED system currently operating at this very narrow band centred around $415 \mathrm{~nm}$ is the Omnilux blue ${ }^{\circledR}$, also from Photo Therapeutics. Although blue light on its own has proved effective in acne treatment, much better and longer-lasting results have been reported with the combination of the blue light for $P$. acnes destruction followed sequentially with $633 \mathrm{~nm}$ red light, which has been historically well-accepted for its anti-inflammatory and wound healing effects. Inflammatory lesion clearance rates at 8-12 weeks after the last treatment session have been reported as ranging from an average of $78 \%$ to around $87 \%$ in acne patients with Burton grades 


\section{ORIGINAL ARTICLES}

from 3 to 5 (Figs. 6,7$){ }^{(12,13)}$

\section{Skin Photorejuvenation}

Skin rejuvenation with light sources is an extremely popular procedure, with patient-driven advances making it a very controversial and 'hot' topic In addition to acne, LED phototherapy has had varying degrees of success in skin rejuvenation as one of the latest phototherapeutic sources to be used in this area. Although ablative laser resurfacing is still the gold standard for skin rejuvenation in severely photo- and chronologically aged skin, it has been associated with severe oedema, crusting and erythema leading to long patient downtime. A new family of phototherapy systems was developed to address this problem by delivering a controlled zone of damage to the upper part of the dermis under an intact epidermis, usually protected by some form of cooling. This would bring into play the range of photoreactions seen in Fig. 1 from mild coagula tion through protein degradation and denaturation to photobiomodulation.

In order to meet the criterion of true skin rejuvenation as defined by Bitter and colleagues, in addition to wrinkles the treatment had to include the entire photoaged skin and associated conditions including elastosis and pigmentary changes. ${ }^{(14)}$ Trelles and colleagues termed this damage type 'delivered thermal damage' (DTD), ${ }^{(15)}$ to differentiate it from the term 'residual thermal damage' (RTD), associated with ablative laser resurfacing and recognized as necessary to stimulate the wound healing processes. ${ }^{(16)}$ Typical systems for NSR included intense pulsed light (IPL) sources, subpurpuric dye lasers, the 1064 and $1320 \mathrm{~nm}$ lines of the Nd:YAG laser, diode lasers $(910 \mathrm{~nm}$ and $1450 \mathrm{~nm})$ and the Er:glas laser $(1540 \mathrm{~nm})$. Many authors agreed that the histological findings with NSR were excellent with all of these modalities, but unfortunately these good dermal architectural improvements were not echoed in the epidermis, ${ }^{(17-23)}$ which remained the 'same old epidermis'. Patient satisfaction was universally much lower than objective clinical assessment. Patient selection and management of patient expectations became extremely important when single modality NSR was used on its own.

The same problem exists to an even greater degree with LED therapy for skin rejuvenation, because by its very nature, LED therapy does not deliver any damage, photothermal or otherwise, to the target tissue. LED therapy on its own cannot hope to tackle deeper lines and wrinkles in the more severely damaged skin. In younger and well-maintained skin, however, with fewer and finer lines and wrinkles, the efficacy is high. In one of the acne studies referenced above, ${ }^{(13)}$ a large percentage of the patients spontaneously reported a better overall skin condition after the study, and objective Mexameter ${ }^{\mathrm{TM}}$ (Courage+Khazaka, Köln, Germany) readings showed statistically significant skin lightening through lower melanin levels, particularly after the $633 \mathrm{~nm}$ treatments.

What is the mechanism(s) behind the success of LED skin rejuvenation? Takezaki et al. previously reported morphological changes in human skin irradiated in vivo with a $633 \mathrm{~nm}$ LED system. ${ }^{(24)}$ No damage was noted in the dermis, but mild perivascular oedema was noted, and it was also clear that, at both a transmission electron microscopic and immunohistochemistry level, $633 \mathrm{~nm}$ LED therapy induced fibroplasia in fibroblasts without any signs of damage in irradiated human skin in vivo, (Fig. 8) $)^{(24)}$ and recruited large numbers of skin-homing t-cells into the irradiated area. ${ }^{(25)}$ After cultivation and semi-quantitative PCR of irradiated skin specimens, with b-actin positive in all subjects for housekeeping, an average count of more than 6000 Th2 and 40 Th3 cells was recorded compared with virtually zero for the unirradiated control specimens. Taking into consideration the existing knowledge that red light at $633 \mathrm{~nm}$ can recruit mast cells into the irradiated area in a mouse tongue model and then accelerate their degranulation, ${ }^{(26)}$ we can hypothesize that this sudden influx of skin-homing Tcells, especially Th2 cells, coupled with the appearance of a large number of proinflammatory substances and cytokines from the accelerated degranulation of mast cells, convinces the skin that it has been, to all intents and purposes, 'wounded', because the inflammatory reaction normally associated with the first phase of wound healing is very clearly in place. Photobiomodulated macrophage cells move to and ingest their targets faster, and release larger quantities of fibroblast growth factor (FGF), ${ }^{(27,28)}$ leaving the matrix in a cleaner and more favourable environment for the photoactivated fibroblasts during the following proliferative wound healing stage. There is in fact no wound, and we refer to this phenomenon as LEDmediated quasi-wounding. The high state of fibroplasia of the irradiated fibroblasts (seen in Fig. 8c above) attests to the fact that they are moving into the proliferative phase, the 2 nd phase of wound healing, without there ever having been a wound.

We can thus argue that LED therapy at $830 \mathrm{~nm}$ and $633 \mathrm{~nm}$ can induce the wound healing process atraumatically and athermally in normal skin, thereby producing well-oriented and more plentiful collagen. The photobiomodulated fibroblasts do not only produce collagen, but also elastin and additionally synthesize ground substance. The combination of these is 


\section{ORIGINAL ARTICLES}

Fig. 6: lue/red LED in the treatment of acne. (a): Before and (b): 12 weeks after treatment in a young female patient with Burton grade 5 acne. Excellent result, no other medication or treatment. (Pictures courtesy of Dr Bruce Russell)
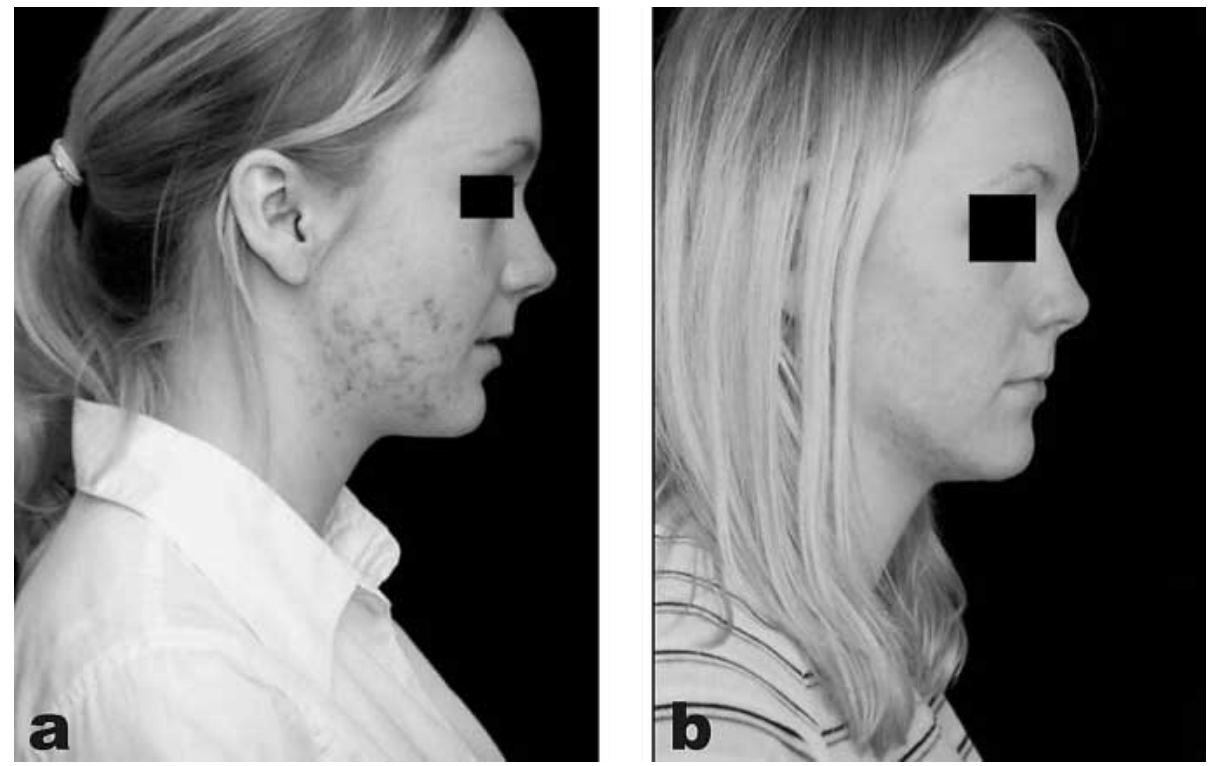

Fig. 7: Blue/red LED in the treatment of acne. (a): Before and (b): 10 weeks after treatment in a young female patient with cystic type acne. A very good result with excellent cosmesis, no other medication or treatment. (Pictures courtesy of Dr Bruce Russell)
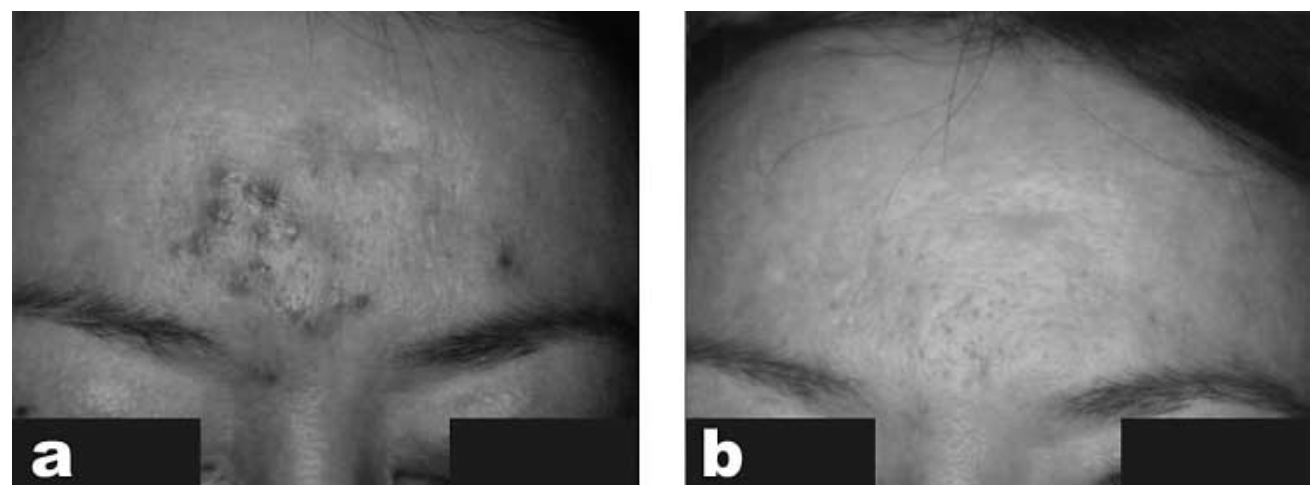

Fig. 8: Transmission electron microscopy showing development of fibroplasia in $633 \mathrm{~nm}$ LED irradiated fibroblasts in a human subject, in vivo. (a): Preirradiation. (b): after 2 sessions, more mitochondria can be seen in the cytoplasm with vimentin fibrils (arrows) increased in number. (c): After 8 sessions, giant electrodense mitochondria are seen, and increasing numbers are appearing by mitosis.A very large amount of vimentin fibrils fill the cytoplasm (arrows). (24)
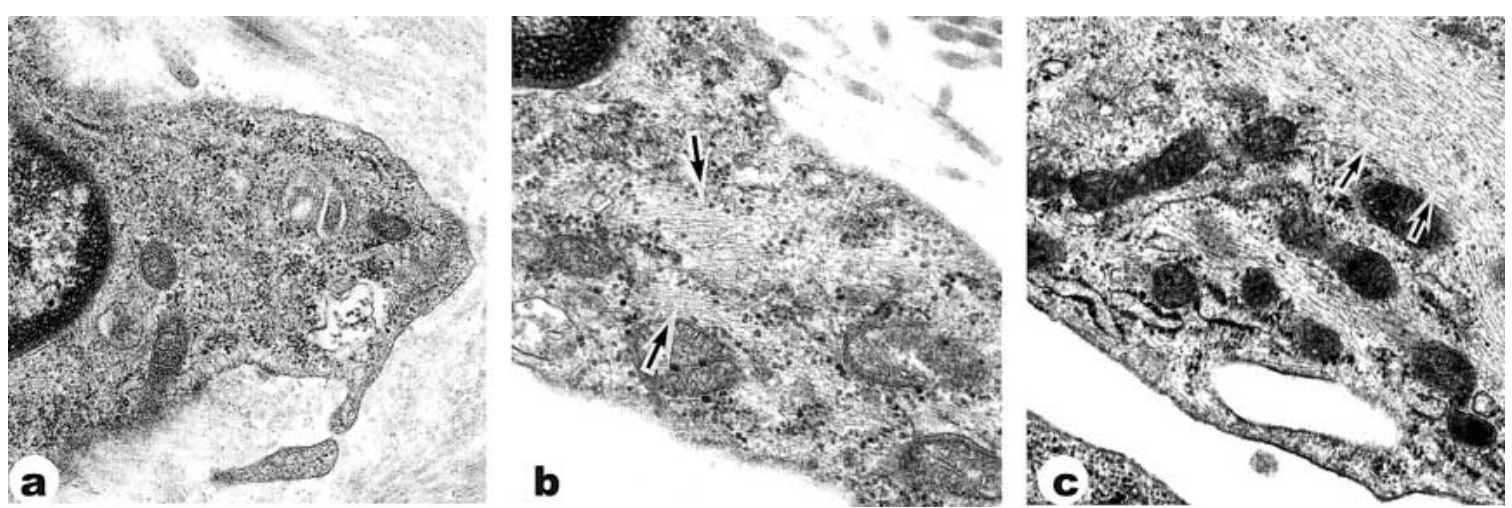
Fig. 9: H \& E Stain of specimens before (left) and 2 weeks after (right) combination $830 \mathrm{~nm} / 633 \mathrm{~nm}$ skin rejuvenation. The development of new collagen fibres is very clear on the right, with a much more compact-looking dermal matrix.
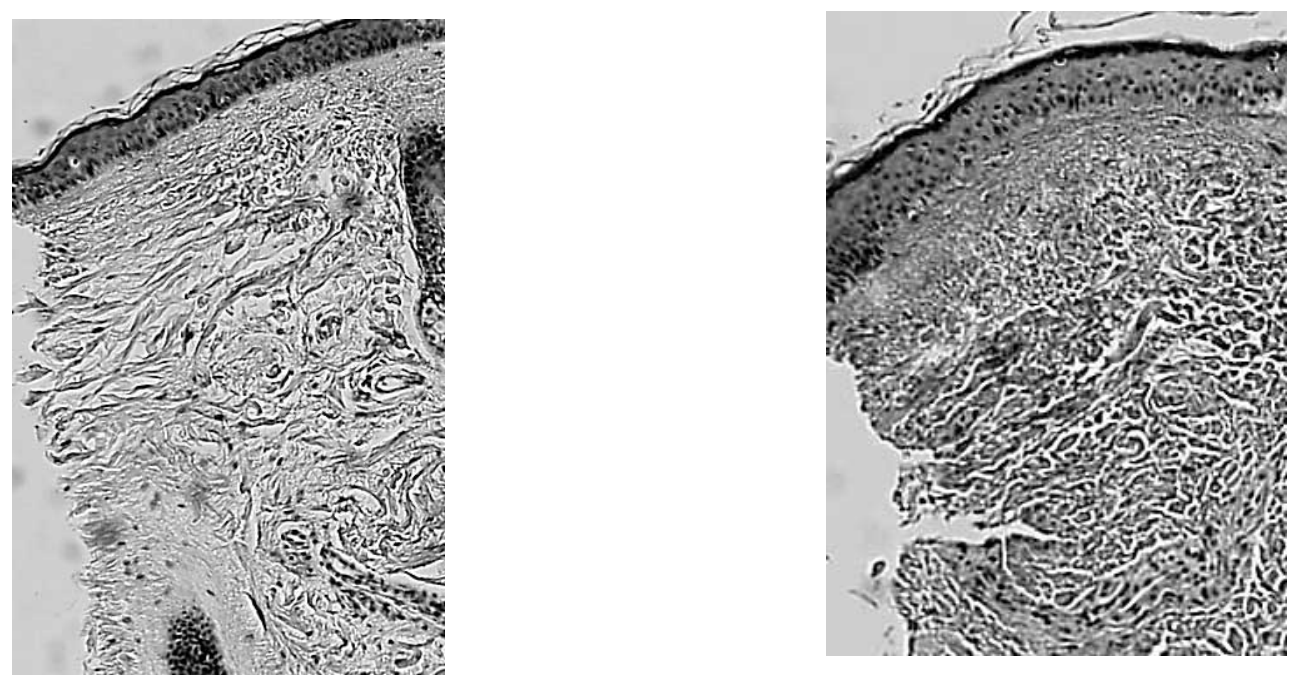

Fig. 10: Elastica van Giesen stain of specimens before (left) and 2 weeks after (right) combination $830 \mathrm{~nm} / 633 \mathrm{~nm}$ skin rejuvenation. The development of new elastin fibres is very clear on the right, particularly in the upper reticular dermis.

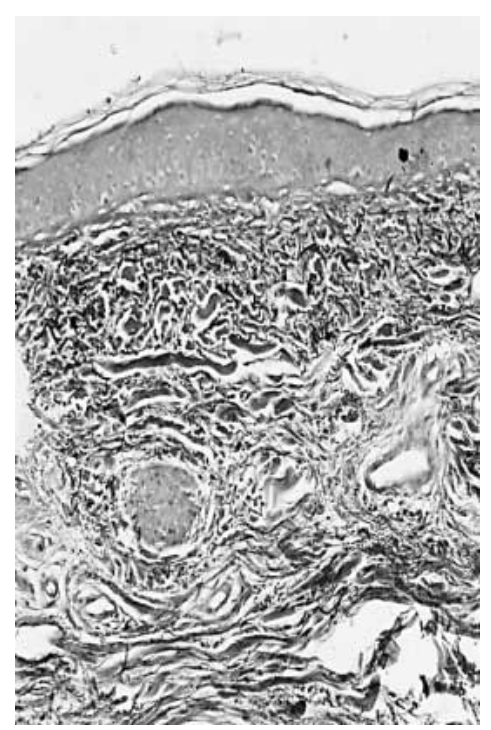

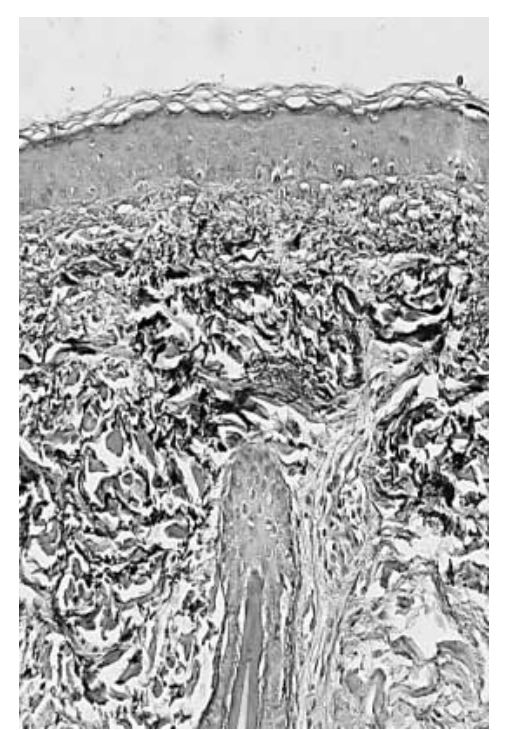

seen as a younger, plumper skin, and histology shows the typical elastotic appearance of the photoaged dermis being replaced by a much better-organized, tighter matrix.(Figs. 9, 10) An additional point of interest based on the increased amount of normal skin-homing $\mathrm{t}$-cells has interesting implications in the treatment of $\mathrm{t}$ cell-related immunomodulated conditions such as acne and psoriasis, using appropriate wavelengths of LED therapy.

The best approach in LED skin rejuvenation has appeared as the combination of near infrared $830 \mathrm{~nm}$ 168 followed by the $633 \mathrm{~nm}$ head, Augmenting this with adjunctive epidermal care should improve the results even better, which has been the case with IPL skin rejuvenation. (29) However, LED therapy might possibly have even more to offer as an adjunctive therapy to conventional and light surgery: this is an area which will yield many interesting findings in the future.

\section{Wound Healing}

The basic principles and the processes of wound healing underpin the uses of LED therapy already men- 
tioned, acne and skin rejuvenation. The inflammatory acne lesion, in addition to its very unpleasant external appearance, also represents a serious dermal wound with disruption of the dermal matrix, which must be encouraged to heal properly to avoid acne scarring. Induction of the wound healing process is essential in skin rejuvenation to help repair the photodamaged dermal matrix. LEDs, however, can be used to accelerate the healing of any kind of wound. LED-enhanced wound healing following a clinical or surgical procedure is a new and exciting field with obvious implications in all fields of clinical practice. The $633 \mathrm{~nm}$ LED array therapy has shown good results post laser assisted blepharoplasty(Fig. 11). (30) Furthermore, 830 $\mathrm{nm} / 633 \mathrm{~nm}$ combination LED therapy has reportedly revitalized the procedure of full face laser ablative resurfacing, cutting healing time by at least half and reducing patient down time dramatically with erythema resolving as early as 3 weeks after a full face resurfacing. (Branson et al, Trelles et al, [submitted]) Wound healing is dramatically accelerated after Er:YAG laser removal of plantar warts. ${ }^{(31)}$

\section{Pain Control}

A large body of data exists on the use of the $830 \mathrm{~nm}$ GaAlAs diode laser for pain attenuation, many of which papers have appeared in this journal. In recent editions, $830 \mathrm{~nm}$ LED therapy has been shown to have excellent promise in sports injury-mediated pain in professional athletes, ${ }^{(32)}$ to remove late postoperative pain following a mastectomy for breast cancer ${ }^{(33)}$ and in relieving pain following laser ablation of plantar warts. ${ }^{(31)}$

\section{Conclusions}

Phototherapy has definitely arrived in the practical clinical field for the treatment of acne, for skin rejuvenation and wound healing, and the new generation of LEDs has emerged as the main phototherapeutic modality used in adjustable, hands-off planar arrays so that patients can receive LED therapy while the clinician or charge nurse looks after other patients, paperwork and so on. Pain control is another exciting aspect of this well-tolerated, pain-free, safe and effective modality. The system already mentioned (Omnilux, from Therapeutics Limited, Fazeley, UK) has been approved by FDA for a variety of applications, including acne, skin rejuvenation and general dermatological applications. LED therapy appears to work best in clinical applications when used in combination: one LED wavelength combined with another, or possibly even better still, LED therapy used in an adjunctive manner to enhance the results obtained with conventional modalities. It is clear that, while LED therapy is not going to turn a poor clinician into a good one, it certainly could help good clinicians achieve even better results, and happier patients.

\section{References}

1: Fubini S (1897): Influenza della luce sulla respirazione del tessuto nervoso. Annali Universali di Medicina e Chirurgia. Serie 1, 250: Fascicolo 7.

2: Calderhead RG. Simultaneous low-reactive level laser therapy (LLLT) in laser surgery: the a-effect explained. In Ohshiro $\mathrm{T}$ and Calderhead RG (Eds) Progress in Laser Therapy. John Wiley and Sons, Chichester, UK. 1991 pp209-214.

3: Ohshiro T (2005): Autosimultaneous laser treatment: a new effect-based classification. Laser Therapy, 14: 11-17.

4: Karu T (1999). Primary and secondary mechanisms of action of visible to near-IR radiation on cells. $J$ Photochem Photobiol B, 49: 1-17.

5: R Glen Calderhead RG (2004): Laser resurfacing today - not all photoscience is photothermal. Journal of Cosmetic Dermatology, 3: 242-243.

6: Calderhead RG, Ohshiro T, Itoh E, Okada T, and Kato Y: The Nd:YAG and GaAlAs lasers; a comparative analysis in pain therapy. In Atsumi $\mathrm{K}$ and Nimsakul N (eds) "Laser Tokyo 81", Section 21 (Laser Acupuncture). Japan Society for Laser Medicine, Tokyo, Japan. 1981. pp 1-4.

7: Whelan HT, Houle JM, Whelan NT et al. (2000): The NASA Light-Emitting Diode Medical ProgramProgress in Space Flight and Terrestrial Applications. Space Tech. \& App. Int'l. Forum. 504: 37-43.

8: Whelan HT, Smits RL, Buchmann EV et al. (2001): Effect of NASA Light-Emitting Diode (LED) Irradiation on Wound Healing. Journal of Clinical Laser Medicine and Surgery, 19: 305-314.

9: Morton CA, and Burden AD (2001): Treatment of multiple scalp basal cell carcinomas by photodynamic therapy. Clin Exp Dermatol, 26: 33-36.

10: Pollock B, Turner D, Stringer MR, Bojar RA, Goulden V, Stables GI, and Cunliffe WJ (2004): Topical aminolaevulinic acid-photodynamic therapy for the treatment of acne vulgaris: a study of clinical efficacy and mechanism of action. British Journal of Dermatology, 151: 616-122.

11: Lowe NJ, and Lowe P (2005): Pilot study to determine the efficacy of ALA-PDT photo-rejuvenation for the treatment of facial ageing. J Cosmet Laser Ther, 7: 159-162 
12: Goldberg DG, Russell B (2006): Combination blue $(415 \mathrm{~nm})$ and red (633 nm) LED phototherapy in the treatment of mild to severe acne vulgaris. J Cos Laser Ther, 8: 71-75.

13: Lee SY, You CE and Park MY (2006): Blue and red light combination LED phototherapy in the treatment of acne vulgaris. Lasers Surg Med, accepted for publication and in press.

14: Bitter PH (2000): Noninvasive rejuvenation of photoaged skin using serial, full-face intense pulsed light treatments. Dermatol Surg, 26: 835-843.

15: Trelles MA, Allones I, Levy JL, Calderhead RG, and Moreno-Arias GA (2004): Combined nonablative skin rejuvenation with the 595- and $1450-\mathrm{nm}$ lasers. Dermatol Surg, 2004 30: 1292-1298.

16: Ratner D, Viron A, Puvión -Dutilleul F, Puvion E (1998): Pilot ultrastructural evaluation of human preauricular skin before and after high-energy pulsed carbon dioxide laser treatment. Arch Dermatol, 134: 582-587.

17: Erickson BD, Kilter SL, Bernstein E, Cozen VA, Dock J et al. (1999): Pulsed dye laser therapy for sun-damaged skin. Lasers Surg Med, 25: 229-236.

18: Bjerring P, Clement M, Heickendorf L, Egevist H and Kiernan M (2000): Selective non- ablative wrinkle reduction by laser. J Cutan Las Ther, 2: 915.

19: Goldberg DJ (2000): New collagen formation after dermal remodeling with an intense pulsed light source. J Cutan Las Ther, 2: 59-61.

20: Goldberg DJ and Cutler KB (2000): Nonablative treatment of rhytids with intense pulsed light. Lasers Surg Med, 26: 196-200.

21: Trelles MA, Allones I and Luna R. (2001): Facial rejuvenation with a nonablative $1320 \mathrm{~nm} \mathrm{Nd:YAG}$ laser: a preliminary clinical and histologic evaluation. Dermatol Surg, 27: 111-116.

22: Alster TS (1995): Improvement of erythematous and hypertrophic scars by the $585 \mathrm{~nm}$ pulsed dye laser. Ann Plast Surg, 32: 186-190.

23: Trelles MA, Allones I and Velez M(2003): Nonablative facial skin photorejuvenation with an intense pulsed light system and adjunctive epider- mal care. Lasers Med Sci, 18: 104-111.

24: Takezaki S, Omi T, Sato S and Kawana S (2005): Ultrastructural observations of human skin following irradiation with visible red light-emitting diodes (LEDs): A preliminary in vivo report. Laser Therapy, 14: 153-160.

25: Takezaki S, Omi T, Sato S, Kawana S (2006): Lightemitting diode phototherapy at $630+/-3 \mathrm{~nm}$ increases local levels of skin-homing T-cells in human subjects. J Nippon Medical School, 73: 7581.

26: Trelles MA, Mayayo E, Miro L, Rigau J and Calderhead RG (1989) The action of LLLT on mast cells: a possible pain mechanism examined. Laser Therapy, 1: 27- 30 .

27: Bolton P.A., Young S. and Dyson M. (1990): Macrophage responsiveness to light therapy - a dose response study. Laser Therapy, 2: 101-106.

28: Bolton P.A., Dyson M. and Young S (1992): The effect of polarized light on the release of growth factors from the U-973 macrophage- like cell line. Laser Therapy, 4: 33-38.

29: Trelles MA, Allones I and Velez M(2003): Nonablative facial skin photorejuvenation with an intense pulsed light system and adjunctive epidermal care. Lasers Med Sci, 18: 104-111.

30: Trelles MA and Allones I (2006): Red light-emitting diode (LED) therapy accelerates wound healing post-blepharoplasty and periocular laser ablative resurfacing. J Cosmet Laser Ther, 8: 39-42.

31: Trelles MA and Calderhead RG (2005): Red lightemitting diode (LED) therapy-assisted healing improves results of Er:YAG laser ablation of plantar verrucae. Laser Therapy, 14: 179-183.

32: Baxter GD, Bleakley C, Glasgow P and Calderhead RG (2005): A near-infrared LED-based rehabilitation system: initial clinical experience. Laser Therapy, 14: 29-36.

33: Trelles MA and Calderhead RG (2005):.Combined infrared laser and LED therapy for postmastectomy pain and discomfort: a case report. Laser Therapy, 14: 41-46. 